\title{
A comprehensive evaluation of the role of genetic variation in follicular lymphoma survival
}

\author{
Fredrik Baecklund ${ }^{1,2^{*}}$, Jia-Nee Foo ${ }^{3}$, Paige Bracci ${ }^{4}$, Hatef Darabi ${ }^{5}$, Robert Karlsson ${ }^{5}$, Henrik Hjalgrim ${ }^{6}$, \\ Richard Rosenquist ${ }^{7}$, Hans-Olov Adami ${ }^{5,8}$, Bengt Glimelius ${ }^{9,10}$, Mads Melbye ${ }^{11}$, Lucia Conde ${ }^{12}$, Jianjun Liư ${ }^{3}$, \\ Keith Humphreys ${ }^{5}$, Christine F Skibola ${ }^{12}$ and Karin E Smedby ${ }^{1,13}$
}

\begin{abstract}
Background: Survival in follicular lymphoma (FL) is highly variable, even within prognostic groups defined by tumor grade and the Follicular Lymphoma International Prognostic Index. Studies suggest that germline single nucleotide polymorphisms (SNPs) may hold prognostic information but further investigation is needed.
\end{abstract}

Methods: We explored the association between SNPs and FL outcome using two approaches: 1) Two independent genome-wide association studies (GWAS) of $~ 300.000$ SNPs followed by a meta-analysis encompassing 586 FL patients diagnosed in Denmark/Sweden 1999-2002 and in the United States 2001-2006; and 2) Investigation of 22 candidate-gene variants previously associated with FL outcome in the Danish/Swedish cohort $(\mathrm{N}=373)$. We estimated time to lymphoma-specific death (approach 1 and 2) and lymphoma progression (approach 2) with hazard ratios (HR) and 95\% confidence intervals (CI) in a multivariable Cox regression model.

Results: In the GWAS meta-analysis, using a random effects model, no variants were associated with lymphoma-specific death at a genome-wide significant level $\left(p<5.0 \times 10^{-8}\right)$. The strongest association was observed for tightly linked SNPs on $17 \mathrm{q} 24$ near the $A B C A 10$ and $A B C A 6$ genes ( $r$ $10491178 \mathrm{HR}_{\text {random }}=3.17,95 \% \mathrm{Cl} 2.09-4.79$, prandom $=5.24 \times 10^{-8}$ ). The $A B C A 10$ and $A B C A 6$ genes belong to a family of genes encoding for $A B C$ transporter proteins, implicated in multidrug resistance. In line with a previous study, rs2466571 in CD46 ( $H R=0.73,95 \% \mathrm{Cl} 0.58-0.91, p=0.006)$ showed nominal association with lymphoma progression, as did two highly linked SNPs in IL8 (rs4073 HR $=0.78,95 \% \mathrm{Cl}$ 0.62-0.97, $\mathrm{p}=0.02$; rs $2227307 \mathrm{HR}=0.75,95 \% \mathrm{Cl} 0.60-0.94, \mathrm{p}=0.01$ ) previously associated with overall survival.

Conclusions: The results suggest a possible role for multidrug resistance in $\mathrm{FL}$ survival and add to the evidence that genetic variation in CD46 and IL8 may have prognostic implications in FL. Our findings need further confirmation in other independent populations or in a larger multicenter GWAS.

Keywords: Follicular lymphoma, Prognosis, Single nucleotide polymorphism, Genome-wide association study, Candidate gene study

\section{Background}

For most patients with follicular lymphoma (FL) the disease is incurable and treatment aims to relieve symptoms and prolong survival [1]. In the clinical setting, established prognostic markers include tumor grade and the Follicular Lymphoma International Prognostic Index (FLIPI) [2]. However, marked variation in outcome remains within

\footnotetext{
* Correspondence: fredrik.baecklund@ki.se

'Department of Medicine Solna, Clinical Epidemiology Unit, Karolinska Institutet, Stockholm, Sweden

${ }^{2}$ Department of Oncology, Karolinska University Hospital Solna, Stockholm, Sweden

Full list of author information is available at the end of the article
}

each risk group [2], and new prognostic markers are therefore desirable for more accurate prognostication and personalized treatment. In the search for such markers, several studies have assessed the impact of inherited genetic variation in FL survival [3-18].

There is growing evidence that the tumor microenvironment and the host immune response, partly determined by host genetics, are important for the clinical course of FL [19]. Germline genetic variation may further affect the metabolism [20] or receptor affinity of anti-cancer drugs [21] with possible implications for prognosis. To date, at least 30 specific germline single 
nucleotide polymorphisms (SNPs), mostly targeting cytokine and immune function genes (e.g., C9, CD46, CD55, CFH, IL2, IL4R, IL8 and FCGR2A), have been associated with FL outcome in previous studies [3,4,6-9,11-18]. However, most studies were small in size $(<150$ FL cases $[3,4,6,8,11-18])$, and the majority of the reported findings are unconfirmed. In recent genome-wide association studies (GWAS), an important role for variation in the human leukocyte antigen (HLA) region has been revealed for risk of FL, and one variant in the HLA class I region has been associated both with FL risk and survival [22-26].

To gain additional knowledge of the role of genetic variation in FL outcome, we explored the association of 300.000 SNPs with lymphoma-specific death in two independent GWAS in Sweden/Denmark [25] and the USA [22], followed by a full meta-analysis encompassing a total of $586 \mathrm{FL}$ cases. We also investigated genetic variants previously reported to be associated with FL survival as well as recently established FL risk-associated SNPs for the association with lymphoma-specific death and progression in the Swedish/Danish $(\mathrm{N}=373)$ and Swedish datasets $(\mathrm{N}=231)$, respectively.

\section{Methods}

This study was approved by the Ethical Review Board in each country (Sweden: The Regional Ethical Review Board in Stockholm; Denmark: Scientific Ethics Committee for the Capital Region of Copenhagen; California, USA: UCSF Human Research Protection Program, Committee on Human Subjects Research) and all study participants gave informed consent.

\section{SCALE study participants}

We included cases with FL in the Scandinavian Lymphoma Etiology (SCALE) study, described in detail elsewhere [27]. Briefly, SCALE is a population-based case-control study of the etiology of malignant lymphomas conducted in Sweden and Denmark 1999 to 2002. Overall, 3,740 incident malignant lymphoma patients 18-74 years of age were included. Through a rapid case ascertainment network, the patients were identified shortly after diagnosis. Lymphoma subtypes were reviewed and reclassified according to the WHO classification [28]. Study participants were restricted to individuals with sufficient knowledge of the Danish or Swedish language to answer questions in a telephone interview and without a history of organ transplantation, HIV infection, or other hematopoietic malignancy. In the study overall, $85 \%$ of eligible NHL patients were included. In Sweden, a detailed registration of subtypes of all eligible patients permitted evaluation of participation rates by NHL subtype, which was $94 \%$ for FL. Early death was an uncommon reason for non-participation among the FL patients (1\%). Four-hundred ninety-nine (85\%) of all interviewed FL patients also gave blood. The median time from diagnosis to venipuncture was three months (interquartile range: one to five months). In SCALE overall, patients who gave blood were similar to all participating patients with regard to age, sex and educational level.

\section{Clinical data and follow-up}

In Sweden, information about established prognostic factors (Ann Arbor stage, performance status, number of involved nodal areas, hemoglobin and lactate dehydrogenase levels), treatments and signs of progression, was collected from medical records. Lymphoma progression was defined as the time to progression, date of start of second-line treatment or lymphoma-specific death, if no second-line treatment was given (data only available in Sweden). Dates and causes of death were obtained through the Cause of Death registry [29], with complete follow-up through February 2, 2012. In Denmark, clinical parameters (Ann Arbor stage, performance status, treatment) and complete follow-up of survival through December 17, 2009, were obtained from the Danish national lymphoma database (LYFO) [30]. Lymphomaspecific death was defined as having lymphoma as the main underlying cause of death. Based on the number of FLIPI risk factors, the Swedish cases were classified as having low, intermediate or high risk of death $(0-1,2$ or $\geq 3$ risk factors, respectively). The Danish patients were classified according to a modified FLIPI based on age $(\geq 60)$ and stage (I-II vs. III-IV) only, as low risk if they had none of these factors, intermediate if they had one, and high risk if they had two risk factors.

\section{Genotyping}

In $400 \mathrm{FL}$ cases for whom a sufficient amount of DNA was available, genotyping of 317,503 SNPs was conducted at the Genome Institute of Singapore within a GWAS using the Illumina HumanHap 300 (version 1.0) array [25]. The resulting dataset was filtered on the basis of genotyping call rates $(\geq 95 \%)$, sample completion rate $(\geq 90 \%)$, minor allele frequency $(\geq 0.03)$ and non-deviation from the Hardy-Weinberg equilibrium $\left(\mathrm{p}<10^{-6}\right)$. SNPs on the $\mathrm{X}$ or $\mathrm{Y}$ chromosomes or with cluster plot problems were excluded. Study participants with gender discrepancies and/or labeling errors were removed, as were samples with evidence of cryptic family relationship (identified using the genome command in PLINK [31]) and population outliers on the basis of their values of the first three principal components (identified using EIGENSTRAT software and unlinked SNPs only [32]). Complete genotyping was obtained for 298,702 SNPs in 373 (94\%) of the genotyped cases.

\section{The University of California San Francisco (UCSF) study} The UCSF study population consisted of 213 FL cases included in a population-based case-control study of 
NHL (>2,055 cases, 2,081 controls) conducted in the San Francisco Bay area of incident NHL at ages 20-84 years 2001 to 2006. The cases were identified through rapid case ascertainment methods by the Cancer Prevention Institute of California with case reporting supplemented by Surveillance, Epidemiology and End Results data [33]. Overall, 69\% of eligible cases participated in the study. Diagnostic material was re-reviewed and classified according to the WHO lymphoma classification by the study pathologist [28]. Blood and/or buccal specimens were collected from $87 \%$ of the cases within median 27 days from diagnosis. Genotyping of 329,294 SNPs was performed using the Illumina HumanCNV370-Duo BeadChip (Illumina, Inc., San Diego, CA). SNPs were excluded for low call rates $(<90 \%)$, low minor allele frequency $(<0.03)$, location on the sex chromosomes or cluster plot problems. Non-European samples according to MDS plot inspection were removed. Vital status and cause of death were updated through August 2012 through record linkage with the Greater Bay Area Cancer registry. Patients were censored at date of death or date of last known contact.

\section{Selection of SNPs associated with FL outcome and established FL risk SNPs}

Published studies of germline polymorphisms and FL outcome were found by searching PubMed and Web of Science (through June 30, 2013). We selected SNPs statistically significantly $(\mathrm{p}<0.05)$ associated with overall or lymphoma-specific survival or with time to progression/ treatment in at least one study and investigated the association of these with lymphoma-specific death and progression in SCALE and the Swedish cohort, respectively. Fifteen studies and 30 SNPs representing 29 separate loci were identified (Table 1). Genotype data on 22 of these markers (21 loci) were available through genotyping $(\mathrm{N}=9)$ or imputation $(\mathrm{N}=13)$. We used 1000 genomes multi-ethnic reference panel and Impute 2 for imputation (http://mathgen.stats.ox.ac.uk/impute/impute_v2. html\#download_reference_data). A strict threshold was set to genotypes with probabilities $>0.9$, SNPs with information scores $>0.8$ and call rates $>0.9$. Six SNPs strongly associated with FL risk in recent GWAS [22-26], of which one (rs6457327 in the HLA class II region) also has been associated with FL prognosis in two studies $[4,18]$, were also selected for survival analysis.

\section{Statistical analysis}

\section{Genome-wide association studies}

In SCALE and UCSF separately, we estimated the association of SNPs with lymphoma-specific death using the Cox proportional hazards model. Time was measured from diagnosis to lymphoma-specific death or end of follow-up. Deaths from other causes than lymphoma were censored. Patients without an event were censored at the time of last known follow-up. The SNPs were coded as 0,1 or 2 based on the number of minor alleles, and treated as continuous variables in the model. Adjustment was made for age at diagnosis, sex and population stratification (using the first three dimensions calculated by using EIGENSTRAT [32] in SCALE and multidimensional scaling [31] in UCSF; in both cohorts unlinked SNPs only were used for these calculations). Data on FLIPI and first-line rituximab was available in SCALE and the Swedish cohort, respectively, but not in UCSF, and hence these covariates were not included in the model. A full meta-analysis of the GWAS results was performed using the DerSimonian-Laird random effects method [34]. PLINK v 1.07 (http://pngu.mgh. harvard.edu/purcell/plink/) [31] and R [35] were used for these analyses. In complementary analyses, top variants in the meta-analysis were also adjusted for FLIPI risk groups (in SCALE) and first-line rituximab (in the Swedish cohort). The top locus in the meta-analysis was further explored in a regional association plot, combining the p-values of association in the meta-analysis, LD data from 1000 Genomes European population, gene information from the UCSC browser, and estimated recombination rates (http://csg.sph.umich.edu/locuszoom/) [36]. The potential functionality of the top SNPs and SNPs in strong LD $\left(r^{2} \geq 0.8\right)$ with these in the 1000 Genomes European population were explored using RegulomeDB, which integrates multiple types of functional data generated by ENCODE and other sources (http://RegulomeDB.org/) [37].

\section{Investigation of SNPs with reported prognostic impact in FL and established FL risk SNPs}

We investigated the association of the selected candidate SNPs and established FL risk SNPs with lymphomaspecific death in SCALE and lymphoma progression in SCALE Sweden using the same Cox model as in the GWAS. The proportional hazard assumption was tested by plotting Schoenfeld residuals against follow-up time; no violations were found. In complementary analyses, associated variants were also adjusted for FLIPI risk groups and first-line rituximab. Also, potential immortal time bias was assessed in the Swedish cohort by starting follow-up at date of venipuncture instead of diagnosis. We further applied the multi-SNP modeling of risk alleles performed in three previous studies (rs5361, rs3799488, rs1799864 and rs1800796 [3]; rs4073, rs2069762, rs3212227 and rs454078 [7]; rs1801131, rs1127717 and rs719235 [15]) on the SCALE dataset. The SNPs were then coded as 0 or 1 , based on the presence of the risk (protective/deleterious) allele $[3,7,15]$. The impact of having one, two or three or more as compared to no risk alleles on lymphoma-specific death was assessed in a Cox model, adjusting for age and sex. Multiple testing was adjusted for with the Bonferroni 
Table 1 List of single nucleotide polymorphisms (SNPs) associated with follicular lymphoma outcome $(p<0.05)$ in at least one previously published study, and results for these variants in other previous studies

\begin{tabular}{|c|c|c|c|c|c|c|c|c|c|}
\hline Chr & Gene & SNP & $\begin{array}{l}\text { Minor/major } \\
\text { allele }\end{array}$ & Study & $\begin{array}{l}\text { \# FL } \\
\text { cases }\end{array}$ & $\begin{array}{l}\text { \# SNPs } \\
\text { tested }\end{array}$ & Outcome & Relative risk ratio & $\begin{array}{l}\text { \# Studies reporting } \\
\text { no association [refs] }\end{array}$ \\
\hline 1 & C1qA & rs172378 & $A / G$ & Racilia 2008 & 133 & 3 & PFS & $2.5(2.0,3.1)$ & none \\
\hline 1 & CD46 & rs2466571 & C/A & Charbonneau 2012 & 107 & 167 & EFS & $1.49(0.86,2.61), p_{\text {trend }}<0.05$ & none \\
\hline 1 & CD55 & rs2564978 & T/C & Charbonneau 2012 & 107 & 167 & EFS & $0.52(0.30,0.88)$ & none \\
\hline 1 & $\mathrm{CFH}$ & rs1065489 & $T / G$ & Charbonneau 2012 & 107 & 167 & EFS & $0.44(0.24,0.81)$ & none \\
\hline 1 & $\mathrm{CFH}$ & rs1329423 & $C / T$ & Charbonneau 2012 & 107 & 167 & EFS & $0.49(0.29,0.82)$ & none \\
\hline 1 & $\mathrm{CFH}$ & rs3766404 & $\mathrm{G} / \mathrm{A}$ & Charbonneau 2012 & 107 & 167 & EFS & $2.25(1.31,3.87)$ & none \\
\hline 1 & CFHR1 & rs436719 & $C / A$ & Charbonneau 2012 & 107 & 167 & EFS & $0.57(0.34,0.96)$ & none \\
\hline 1 & CFHR5 & rs6694672 & $\mathrm{G} / \mathrm{T}$ & Charbonneau 2012 & 107 & 167 & EFS & $2.63(1.41,4.92)$ & none \\
\hline \multirow[t]{2}{*}{1} & FCGR2A & rs1801274 & $A / G$ & Weng 2003 & 87 & 2 & PFS & Decreased rate, Plog-rank $<0.02$ & $4[10,13,14,16]$ \\
\hline & & & $A / G$ & Cerhan 2007 & 278 & 73 & OS & $0.58(0.31,1.04), p_{\text {trend }}=0.04$ & $2[10,13]$ \\
\hline \multirow[t]{4}{*}{1} & FCGR3A & rs396991 A & $\mathrm{G} / \mathrm{T}$ & Weng 2003 & 87 & 2 & PFS & Decreased rate, Plog-rank $<0.03$ & $5[6,10,13,14,16]$ \\
\hline & & & $\mathrm{G} / \mathrm{T}$ & Ghielmini 2005 & 185 & 1 & EFS & $0.5(0.3,0.9)$ & $1[5]$ \\
\hline & & & $\mathrm{G} / \mathrm{T}$ & Persky 2012 & 142 & 2 & OS & $0.33(0.11 ; 0.96)$ & $1[10]$ \\
\hline & & & $\mathrm{G} / \mathrm{T}$ & Cartron 2002 & 49 & 2 & RR & $1.5(1.2,1.9)$ & $1[10]$ \\
\hline 1 & MTHFR & rs1801131 & $C / A$ & Wang 2009 & 192 & 66 & OS & $2.00(1.04,3.84)$ & none \\
\hline 1 & SELE & rs5361 & & Aschenbrook- Kilfoy 2012 & 117 & 82 & OS & $0.10(0.02,0.48)$ & $1[7]$ \\
\hline 2 & ILIRN & rs454078 & T/A & Cerhan 2007 & 278 & 73 & OS & $0.50(0.28,0.87)$ & $1[3]$ \\
\hline 3 & CCR2 & rs1799864 ${ }^{\mathrm{A}}$ & & Aschenbrook- Kilfoy 2012 & 117 & 82 & OS & $0.27(0.08,0.86)$ & $1[7]$ \\
\hline 3 & FTHFD & rs1127717 & $\mathrm{G} / \mathrm{A}$ & Wang 2009 & 192 & 66 & OS & $1.99(1.07,3.7)$ & none \\
\hline 4 & IL2 & rs2069762 & $\mathrm{G} / \mathrm{T}$ & Cerhan 2007 & 278 & 73 & OS & $1.81(1.06,3.07)$ & none \\
\hline \multirow[t]{2}{*}{4} & IL8 & rs4073 & T/A & Aschenbrook- Kilfoy 2012 & 117 & 82 & OS & $2.60(1.10,6.15)$ & none \\
\hline & & & $A / T$ & Cerhan 2007 & 278 & 73 & OS & $0.47(0.27,0.79), p_{\text {trend }}=0.06$ & none \\
\hline \multirow[t]{2}{*}{4} & IL8 & rs2227307 & T/G & Aschenbrook- Kilfoy 2012 & 117 & 82 & OS & $2.57(1.07,6.17)$ & none \\
\hline & & & $\mathrm{G} / \mathrm{T}$ & Cerhan 2007 & 278 & 73 & OS & $0.53(0.31,0.91), p_{\text {trend }}=0.11$ & none \\
\hline 4 & IRF2 & rs $3775567^{A}$ & $\mathrm{~T} /$ & Gibson $2012^{A}$ & 244 & 6679 & OS & $3.18(1.72,5.87)$ & none \\
\hline 5 & C9 & rs1421094 & $A / G$ & Charbonneau 2012 & 107 & 167 & EFS & $0.54(0.32,0.90)$ & none \\
\hline 5 & IL12B & rs3212227 & $C / A$ & Cerhan 2007 & 278 & 73 & OS & $2.01(1.18,3.42)$ & none \\
\hline \multirow[t]{2}{*}{6} & C6orf15 & rs6457327 & $A / C$ & Wrench 2011 & 218 & 2 & $\pi T$ & 2.25. $(1.16,4.36)$ & none \\
\hline & & & & Berglund 2011 & 102 & 1 & OS & Increased rate, $p=0.006$ & none \\
\hline 6 & IFNGR1 & rs3799488 & & Aschenbrook- Kilfoy 2012 & 117 & 82 & OS & $3.19(1.09,9.34)$ & $1[7]$ \\
\hline 8 & GGH & rs719235 & $T / G$ & Wang 2009 & 192 & 66 & OS & $2.49(1.21,5.14)$ & none \\
\hline
\end{tabular}


Table 1 List of single nucleotide polymorphisms (SNPs) associated with follicular lymphoma outcome $(p<0.05)$ in at least one previously published study, and results for these variants in other previous studies (Continued)

\begin{tabular}{|c|c|c|c|c|c|c|c|c|c|}
\hline 9 & GALNT12 & rs10987898 ${ }^{\mathrm{A}}$ & $\mathrm{G} /$ & Gibson $2012^{A}$ & 244 & 6679 & OS & $0.48(0.27,0.83)$ & none \\
\hline 9 & GALNT12 & rs10819377 & $\mathrm{T} /$ & Gibson $2012^{\mathrm{A}}$ & 244 & 6679 & OS & $0.62(0.36,1.03), p_{\text {trend }}<0.001$ & none \\
\hline 11 & CXCR5 & rs1790192 & $\mathrm{G} / \mathrm{A}$ & Charbonneau 2013 & 172 & 10 & EFS & $0.64(0.47 ; 0.87)$ & none \\
\hline 16 & IL4R & rs1801275 & & Aschenbrook- Kilfoy 2012 & 117 & 82 & OS & $2.35(1.07,5.19)$ & none \\
\hline 20 & BMP7 & rs $6025446^{A}$ & $\mathrm{G} / \mathrm{A}$ & Gibson $2012^{\mathrm{A}}$ & 244 & 6679 & OS & $0.41(0.21,0.69)$ & none \\
\hline 22 & MIF & rs755622 & & Aschenbrook- Kilfoy 2012 & 117 & 82 & OS & $2.45(1.09,5.47)$ & none \\
\hline
\end{tabular}

$\mathrm{EFS}=$ event-free survival, $\mathrm{PFS}=$ progression-free survival, $\mathrm{OS}=$ overall survival, $\mathrm{TT}=$ time to transformation, $\mathrm{RR}=$ response to Rituximab. $\mathrm{R}=\mathrm{Rituximab}, \mathrm{KM}=$ Kaplan-Meier curve. ${ }^{\mathrm{A} V a r i a n t}$ was not available for evaluation in the present study. 
method [38]. The analyses were done in SAS 9.2. Power calculations were performed in R (using survSNP) [35].

\section{Results}

\section{Patient characteristics}

The Danish-Swedish FL cohort was followed for a median of 8.9 years from diagnosis (range 4.3 months to 12.1 years). Median age at diagnosis was 57 years and $65 \%$ had Ann Arbor stage III or IV disease (Table 2). One-hundred thirty-seven patients (37\%) died of which 88 deaths $(64 \%)$ were classified as lymphoma-specific.
The UCSF cohort was followed for a median of 7.5 years (range 6.9 months to 10.3 years). Twenty-six percent $(\mathrm{N}=56)$ died, of which $43 \%(\mathrm{~N}=24)$ were classified as lymphoma-specific deaths. In Swedish patients, progression occurred in 67\% $(\mathrm{N}=155)$, and rituximab was used in first-line treatment in $10 \%$ and overall in $47 \%$. Overall survival differed as expected between risk groups defined by FLIPI (low, intermediate, high; Plog-rank $<0.05)$ in the total SCALE study population and in the Swedish and Danish populations separately (data not shown).

Table 2 Characteristics of the study participants with follicular lymphoma in the Scandinavian Lymphoma Etiology (SCALE) study (Denmark/Sweden) and in the University of California San Francisco (UCSF) study

\begin{tabular}{|c|c|c|c|c|}
\hline & SCALE overall N (\%) & Swedish FL cases N (\%) & Danish FL cases N (\%) & UCSF FL cases $\mathbf{N}(\%)$ \\
\hline Number of participants & 373 & $231(62)$ & $142(38)$ & 213 \\
\hline Sex (men) & $185(50)$ & $111(48)$ & $74(52)$ & $110(52)$ \\
\hline \multicolumn{5}{|l|}{ Age (years) } \\
\hline $18-34$ & $9(2)$ & $6(3)$ & $2(1)$ & $3(1)$ \\
\hline $35-44$ & $30(8)$ & $15(6)$ & $13(9)$ & $15(7)$ \\
\hline $45-54$ & $105(27)$ & $58(25)$ & $43(30)$ & $50(23)$ \\
\hline $55-64$ & $139(36)$ & $83(36)$ & $53(37)$ & $73(34)$ \\
\hline $65-75$ & $100(26)$ & $69(30)$ & $31(22)$ & $42(20)$ \\
\hline $76-84$ & - & - & - & $30(14)$ \\
\hline Median (range) & $57(22-74)$ & $58(22-74)$ & $56(33-72)$ & $60(29-84)$ \\
\hline Participant born in Sweden or Denmark (yes) & $342(92)$ & $203(88)$ & $139(98)$ & - \\
\hline Parents born in Sweden or Denmark (yes) & $331(89)$ & $193(84)$ & $138(97)$ & - \\
\hline Participants of European ancestry ${ }^{B}$ & - & - & - & $213(100)$ \\
\hline \multicolumn{5}{|l|}{ Ann Arbor stage } \\
\hline । & $77(22)$ & $50(23)$ & $27(19)$ & - \\
\hline$\|$ & $48(13)$ & $32(15)$ & $16(12)$ & - \\
\hline III & $100(28)$ & $69(32)$ & $31(22)$ & - \\
\hline IV & $133(37)$ & $68(31)$ & $65(47)$ & - \\
\hline Missing & 15 & 13 & 2 & - \\
\hline \multicolumn{5}{|l|}{ FLIPI $^{\mathrm{A}}$} \\
\hline Low risk & $136(36)$ & $107(46)$ & $29(20)$ & - \\
\hline Intermediate risk & $152(41)$ & $68(29)$ & $84(59)$ & - \\
\hline High risk & $85(23)$ & $56(24)$ & $29(20)$ & - \\
\hline Progression (yes) & - & $155(67)$ & - & - \\
\hline Missing & - & $2(1)$ & - & - \\
\hline Transformation (yes) & - & $56(26)$ & - & - \\
\hline Missing & - & $12(5)$ & - & - \\
\hline Rituximab first line/any treatment line (yes) & - & $24(10) / 106(47)$ & - & - \\
\hline Missing & - & $5(2)$ & - & - \\
\hline Died & $137(37)$ & $88(38)$ & $49(35)$ & $56(26)$ \\
\hline Died due to lymphoma & $88(24)$ & $54(23)$ & $34(24)$ & $24(11)$ \\
\hline
\end{tabular}

${ }^{\mathrm{A}}$ The Swedish patients were classified according to the number of FLIPI risk factors. The Danish patients were classified according to a modified FLIPI, based on age and stage only.

${ }^{B}$ Non-European samples according to MDS plot inspection were removed in UCSF. 


\section{Genome-wide association studies and meta-analysis}

In SCALE and UCSF separately, no variants were associated with lymphoma-specific death at a genome-wide significant level $\left(\mathrm{p} \leq 5.0 \times 10^{-8}\right)$. Thirteen variants in SCALE (on chromosomes 1, 4, 8 and 17; Additional file 1: Figure S1a) and five variants in UCSF (on chromosomes 1, 2, 6, 9 and 20; Additional file 1: Figure S1b) were associated with lymphoma-specific death with a $\mathrm{p} \leq 10^{-6}$, which was more than would be expected by chance (Additional file 1: Figure S2a and S2b).

In the meta-analysis of the 298,702 variants in SCALE and 319,693 in UCSF, 295,134 variants were present in both cohorts. No variant reached genome-wide significant level of association $\left(\mathrm{p}<5.0 \times 10^{-8}\right)$. The strongest signals were observed for SNPs on chromosome 1, 4, 17 and 19 (Additional fle 1: Figure S3), for which the p-values were smaller than would be expected by chance (Additional file 1: Figure S4). The strongest association with lymphoma-specific death was observed at rs10491178 $\left(\mathrm{HR}_{\text {random }}=3.17,95 \%\right.$ CI 2.09-4.79, p $\left._{\text {random }}=5.24 \times 10^{-8}\right)$, located on $17 \mathrm{q} 24$ in the ATP binding cassette A10 gene (ABCA10) (Table 3). An additional five SNPs on 17q24, close to $A B C A 6$, showed similar associations and were in strong linkage disequilibrium (LD) with $\mathrm{rs} 10491178\left(\mathrm{r}^{2} \geq\right.$ 0.96 in the SCALE cohort; Additional file 1: Figure S5). One SNP on chromosome 1 showed a suggestive association of prandom $=10^{-7}\left(\mathrm{rs} 3131729 \mathrm{HR}_{\text {random }}=2.45,95 \% \mathrm{CI}\right.$ 1.75-3.44, prandom $=2.22 \times 10^{-7}$; Table 3). Another SNP on chromosome 1 showed similar association and was linked with rs3131729 $\left(\mathrm{r}^{2}=0.7\right)$ (Additional file 1: Table S1). One intergenic variant on chromosome 4 (rs11932201 $\mathrm{HR}_{\text {random }}=2.16,95 \%$ CI $1.57-2.97, \mathrm{p}_{\text {random }}=2.24 \times 10^{-6}$ ) and one variant located on 19q13 (rs2250066 $\mathrm{HR}_{\text {random }}=$ 2.11, 95\% CI 1.52-2.94, prandom $=9.38 \times 10^{-6}$ ) also showed suggestive associations at $\mathrm{p}_{\text {random }}=10^{-6}$ (Table 3). For these ten top SNPs, there was no indication of heterogeneity between the studies ( $p_{\text {heterogeneity }}>0.05, \mathrm{I}^{2}=0$; Additional file 1: Table S1).

Among the top 48 SNPs in the UCSF GWAS with $\mathrm{p} \leq 10^{-5}$ seven were not genotyped in SCALE. We were able to impute six of these with high confidence (information score $>0.8$ ). The meta-analysis of these SNPs gave no additional suggestive associations (data not shown).

There was no correlation between rs10491178 and performance status, lactate dehydrogenase level (Swedish cohort, $\mathrm{N}=231$ ) or FLIPI risk groups (SCALE cohort, $\mathrm{N}=373$; Fisher's Exact test $\mathrm{p} \geq 0.15$; data not shown). For rs10491178, rs3131729, rs11932201 and rs2250066, additional adjustment for FLIPI and first-line rituximab in SCALE and the Swedish cohort, respectively, did not alter the results meaningfully (data not shown). The association of the top SNP at 17q24 with overall survival (SCALE) was weaker compared with lymphoma-specific death $\left(\mathrm{rs} 10491178 \mathrm{HR}=2.00,95 \% \mathrm{CI} 1.35-2.97, \mathrm{p}=5.68 \times 10^{-4}\right)$, and there was no association with lymphoma progression (Swedish cohort; Additional file 1: Table S2).

Combining data in the RegulomeDB suggested a putative functional role for six of the ten top SNPs, of which rs10491178 (chr 17), rs11932201 (chr 4) and rs2250066 (chr 19) had RegulomeDB scores of 5, indicating that they may affect transcription factor binding or are located in a DNAse hypersensitivity site. There was no data for rs3131729 (chr 1). The lowest RegulomeDB score (i.e. strongest evidence of being in a regulatory site) among our top SNPs and highly linked variants $\left(\mathrm{r}^{2} \geq 0.8\right)$ was found at the SNP rs113464685 in ABCA10 (score 3b), in LD with rs10491178 (data not shown). After imputing the genotypes for rs113464685 in SCALE (as described in Methods for the candidate gene SNPs), we ran the Cox regression for this SNP and found estimates for lymphoma-specific death virtually identical to those of the other SNPs on $17 \mathrm{q} 24 \quad(\mathrm{HR}=3.10,95 \% \mathrm{CI}$ 1.98-4.89, $\left.\mathrm{p}=1.12 \times 10^{-6}\right)$.

\section{Investigation of SNPs with reported prognostic impact in FL and established FL risk SNPs}

For lymphoma-specific death (SCALE cohort, $\mathrm{N}=373$ ), we had $\geq 80 \%$ power to detect $H R \geq 2.0$ (or $\leq 0.5$ ) for rare variants (minor allele frequency $(\mathrm{MAF})=0.09$ [8]) and $\mathrm{HR} \geq 1.5$ (or $\leq 0.67$ ) for more common variants (MAF = $0.49)$ (Additionl file 1: Figure S6). For lymphoma progression (Swedish cohort, $\mathrm{N}=231$ ), we had $\geq 80 \%$ power to detect an $H R \geq 1.75$ (or $\leq 0.57$ ) for variants with MAF $\geq 0.09$. Among the 22 candidate SNPs evaluated, two imputed variants showed nominally significant association $(\mathrm{p}<0.05)$ with lymphoma-specific death: rs1801131 in MTHFR $(\mathrm{HR}=0.69,95 \%$ CI 0.49-0.97, $\mathrm{p}=0.03)$ and $\mathrm{rs} 2069762$ in $I L 2(\mathrm{HR}=0.63,95 \% \mathrm{CI}$ $0.43-0.92, \mathrm{p}=0.02$; Table 4). rs1801131 was also associated with lymphoma progression $(\mathrm{HR}=0.59,95 \% \mathrm{CI}$ 0.45-0.77, $\mathrm{p}=0.0001)$. The direction of association for rs1801131 and rs2069762 with the two lymphomaspecific outcomes was opposite of what was previously reported for overall survival (Table 1) $[7,15]$. In line with a previous study investigating event-free survival in FL [8], we found that the C allele of rs2466571 in the CD46 gene was associated with shorter time to lymphoma progression $(\mathrm{HR}=1.37,95 \%$ CI $1.10-1.72$, $\mathrm{p}=0.006$; Table 4). Likewise, the estimates for two imputed tightly linked SNPs $\left(\mathrm{r}^{2}=0.99\right)$ in the IL8 gene, rs4073 and rs2227307 $\left(\mathrm{HR}_{\mathrm{rs} 4073}=0.78\right.$, 95\% CI 0.62$0.97, \mathrm{p}=0.02 ; \mathrm{HR}_{\mathrm{rs} 2227307}=0.75,95 \%$ CI $0.60-0.94, \mathrm{p}=$ 0.01 ), and lymphoma progression were in the same direction as in two previous studies investigating overall survival in FL (Table 4) [3,7]. Additional adjustment with FLIPI categories or first-line rituximab only marginally altered these results (data not shown). Starting follow-up at date of venipuncture instead of diagnosis 
Table 3 Top single-nucleotide polymorphisms (SNPs) associated with lymphoma-specific death ${ }^{A}\left(p_{\text {random }} \leq 10^{-6}\right)$ in the meta-analysis of FL patients in SCALE and UCSF $(\mathrm{N}=586)$

\begin{tabular}{|c|c|c|c|c|c|c|c|c|c|c|c|c|c|c|c|c|}
\hline \multirow[b]{2}{*}{ Chr } & \multirow[b]{2}{*}{ SNP } & \multirow[b]{2}{*}{ Position } & \multirow[b]{2}{*}{ A1 } & \multirow[b]{2}{*}{ A2 } & \multirow[b]{2}{*}{ MAF } & \multirow{2}{*}{$\begin{array}{l}\text { SCALE } \\
\text { HR }(95 \% \mathrm{Cl})\end{array}$} & \multirow[b]{2}{*}{$p_{\text {SCALE }}$} & \multirow{2}{*}{$\begin{array}{l}\text { UCSF } \\
\text { HR }(95 \% \mathrm{Cl})\end{array}$} & \multirow[b]{2}{*}{ PucsF } & \multirow{2}{*}{$\begin{array}{l}\text { Meta-analysis } \\
\text { HR }(95 \% \mathrm{Cl})\end{array}$} & \multirow[b]{2}{*}{ p RANDOM } & \multicolumn{2}{|c|}{ Heterogeneity } & \multirow[b]{2}{*}{ Gene } & \multirow[b]{2}{*}{ Left gene } & \multirow[b]{2}{*}{ Right gene } \\
\hline & & & & & & & & & & & & $\mathrm{p}_{\text {HET }}$ & $1^{2}$ & & & \\
\hline 17 & rs10491178 & 64661568 & A & G & 0.06 & $3.10(1.97 ; 4.89)$ & $1.13 \mathrm{E}-06$ & $3.50(1.28 ; 9.53)$ & $1.36 \mathrm{E}-02$ & $3.17(2.09 ; 4.79)$ & 5.24E-08 & 0.83 & 0 & $\mathrm{ABCA} 10$ & ABCA6 & LOC100133319 \\
\hline 1 & rs3131729 & 57971355 & A & G & 0.13 & $2.58(1.77 ; 3.76)$ & $9.24 \mathrm{E}-07$ & $2.00(0.92 ; 4.31)$ & $7.58 \mathrm{E}-02$ & $2.45(1.75 ; 3.44)$ & $2.22 \mathrm{E}-07$ & 0.56 & 0 & DAB1 & $\mathrm{C} 8 \mathrm{~B}$ & LOC729423 \\
\hline 4 & rs11932201 & 14304530 & C & A & 0.17 & $2.10(1.48 ; 2.97)$ & $2.93 \mathrm{E}-05$ & $2.50(1.13 ; 5.55)$ & 2.29E-02 & $2.16(1.57 ; 2.97)$ & 2.24E-06 & 0.69 & 0 & NA & LOC152742 & LOC441009 \\
\hline 19 & rs 2250066 & 56220931 & A & G & 0.15 & $2.07(1.45 ; 2.97)$ & $7.31 \mathrm{E}-05$ & $2.35(1.01 ; 5.44)$ & 4.40E-02 & $2.11(1.52 ; 2.94)$ & $9.38 \mathrm{E}-06$ & 0.79 & 0 & KLK11 & KLK10 & KLK12 \\
\hline
\end{tabular}

A2 = major allele, MAF = minor allele frequency, SCALE = Scandinavian lymphoma etiology study, UCSF = University of California, San Francisco, NA = not applicable.

AEstimated with Hazard ratio, HR, and $95 \%$ confidence interval, $\mathrm{Cl}$, adjusting for age at diagnosis and three principal components.

BMAF calculated in SCALE.

Results are sorted by random effects $\mathrm{p}$-value. The minor allele (A1) was investigated for association. 
Table 4 Relative risk ${ }^{A}$ of lymphoma-specific death and lymphoma progression for selected SNPs previously associated with any follicular lymphoma outcome in at least one previous study

\begin{tabular}{|c|c|c|c|c|c|c|c|c|c|c|}
\hline \multirow[b]{2}{*}{ Chr } & \multirow[b]{2}{*}{ Gene } & \multirow[b]{2}{*}{ SNP } & \multirow[b]{2}{*}{ Position } & \multirow[b]{2}{*}{$\mathrm{A} 1$} & \multirow[b]{2}{*}{$\mathrm{A} 2$} & \multirow[b]{2}{*}{ MAF } & \multicolumn{2}{|c|}{ Lymphoma-specific death } & \multirow{2}{*}{$\begin{array}{l}\text { Progression }^{\mathrm{B}} \\
\text { HR }(95 \% \mathrm{Cl})^{\mathrm{A}}\end{array}$} & \multirow[b]{2}{*}{$p$-value } \\
\hline & & & & & & & $\mathrm{HR}(95 \% \mathrm{Cl})^{\mathrm{A}}$ & $\mathrm{p}$-value & & \\
\hline 1 & CD46 & rs2466571 & 206006669 & A & C & 0.49 & $0.98(0.73 ; 1.32)$ & 0.90 & $1.37(1.10 ; 1.72)$ & 0.006 \\
\hline 1 & CD55 & rs $2564978^{c}$ & 205561039 & $\mathrm{~T}$ & C & 0.31 & $0.88(0.62 ; 1.25)$ & 0.47 & $1.13(0.86 ; 1.49)$ & 0.38 \\
\hline 1 & CFH & rs1065489 & 194976397 & A & C & 0.15 & $0.89(0.57 ; 1.39)$ & 0.60 & $0.92(0.65 ; 1.31)$ & 0.66 \\
\hline 1 & $\mathrm{CFH}$ & rs $1329423^{C}$ & 194913010 & C & T & 0.22 & $0.94(0.66 ; 1.39)$ & 0.81 & $1.06(0.79 ; 1.42)$ & 0.71 \\
\hline 1 & $\mathrm{CFH}$ & rs3766404 & 194918455 & G & A & 0.16 & $1.14(0.77 ; 1.68)$ & 0.51 & $1.12(0.80 ; 1.55)$ & 0.52 \\
\hline 1 & CFHR5 & rs6694672 & 195212412 & G & T & 0.09 & $1.01(0.61 ; 1.67)$ & 0.97 & $1.02(0.66 ; 1.57)$ & 0.93 \\
\hline 1 & FCGR2A & rs1801274 & 159746369 & A & G & 0.47 & $0.76(0.56 ; 1.03)$ & 0.08 & $0.86(0.68 ; 1.08)$ & 0.19 \\
\hline 1 & MTHFR & rs1801131 c & 11777063 & G & T & 0.32 & $0.69(0.49 ; 0.97)$ & 0.03 & $0.59(0.45 ; 0.77)$ & $0.0001^{\mathrm{E}}$ \\
\hline 1 & SELE & rs5361 & 167967684 & C & A & 0.11 & $1.04(0.64 ; 1.69)$ & 0.89 & $0.90(0.61 ; 1.32)$ & 0.59 \\
\hline 2 & ILIRN & rs $454078^{c}$ & 113605264 & $\mathrm{~T}$ & A & 0.28 & $0.99(0.69 ; 1.42)$ & 0.96 & $0.94(0.72 ; 1.23)$ & 0.66 \\
\hline 3 & FTHFD & rs $1127717^{C}$ & 127308749 & C & T & 0.16 & $0.91(0.58 ; 1.43)$ & 0.69 & $0.98(0.70 ; 1.38)$ & 0.92 \\
\hline 4 & $1 L 2$ & rs2069762 & 123597430 & C & $A$ & 0.26 & $0.63(0.43 ; 0.92)$ & 0.02 & $0.89(0.69 ; 1.16)$ & 0.38 \\
\hline 4 & IL8 & rs $4073^{c}$ & 74824888 & A & $\mathrm{T}$ & 0.46 & $0.93(0.70 ; 1.25)$ & 0.64 & $0.78(0.62 ; 0.97)$ & 0.02 \\
\hline 4 & IL8 & rs $2227307^{C}$ & 74825533 & G & T & 0.45 & $0.93(0.69 ; 1.25)$ & 0.63 & $0.75(0.60 ; 0.94)$ & 0.01 \\
\hline 5 & C9 & rs1421094 & 39391348 & A & G & 0.37 & $1.19(0.88 ; 1.61)$ & 0.27 & $1.00(0.79 ; 1.27)$ & 0.98 \\
\hline 5 & IL $12 B$ & rs $3212227^{c}$ & 158675528 & G & T & 0.17 & $1.21(0.81 ; 1.80)$ & 0.36 & $1.42(1.03 ; 1.94)$ & 0.03 \\
\hline 6 & C6orf15 & rs6457327 & 31182009 & A & C & 0.42 & $0.90(0.66 ; 1.24)$ & 0.53 & $0.90(0.71 ; 1.14)$ & 0.38 \\
\hline 6 & IFNGRI & rs3799488 & 137561473 & G & A & 0.12 & $0.76(0.46 ; 1.24)$ & 0.27 & $1.12(0.79 ; 1.57)$ & 0.53 \\
\hline 8 & GGH & rs $719235^{c}$ & 64114235 & A & C & 0.32 & $0.96(0.69 ; 1.34)$ & 0.82 & $1.00(0.77 ; 1.29)$ & 0.99 \\
\hline 9 & GALNT12 & rs10819377 & 100643533 & A & G & 0.46 & $0.76(0.56 ; 1.03)$ & 0.07 & $0.89(0.71 ; 1.12)$ & 0.33 \\
\hline 16 & IL4R & rs1801275 & 27281901 & G & A & 0.25 & $0.91(0.64 ; 1.30)$ & 0.62 & $0.85(0.65 ; 1.12)$ & 0.26 \\
\hline 22 & MIF & rs $755622^{c}$ & 22566392 & C & G & 0.16 & $0.76(0.49 ; 1.16)$ & 0.20 & $0.85(0.61 ; 1.18)$ & 0.34 \\
\hline
\end{tabular}

${ }^{A}$ Estimated with Hazard ratio, $\mathrm{HR}$, and $95 \%$ confidence interval, $\mathrm{Cl}$. Adjusted for age at diagnosis, sex and three principal components.

${ }^{B}$ Swedish cases only $(n=231)$ for time to progression.

CImputed SNP.

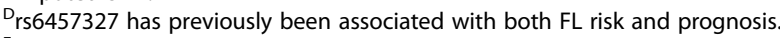

EOpposite direction of association to previous study [15].

The minor allele (A1) was investigated for association except for rs2466571 (CD46), where the major allele (A2) was used as reference for easier comparison with previous study result.

also did not change the results (data not shown). After correcting for performing 54 tests (27 SNPs and two outcomes), the association of rs1801131 (MTHFR) with lymphoma progression remained significant (Bonferroni corrected $\mathrm{p}=0.01$ ).

We finally stratified patients by the number of deleterious/protective alleles, as defined in three previous studies $[3,7,15]$, but found no significant associations with the outcomes in the present study (data not shown). None of the six SNPs previously reported to be associated with risk of FL [22-26] were significantly associated with lymphomaspecific death or progression (Table 4 (rs6457327) and Additional file 1: Table S3).

\section{Discussion}

In this GWAS meta-analysis of common genetic variation in FL prognosis in a total of 586 patients, we did not identify any variants associated with lymphoma- specific death at a genome-wide significant level $\left(\mathrm{p}<5.0 \times 10^{-8}\right)$. The strongest association was observed for tightly linked SNPs located on 17q24 near the $A B C A 10$ and $A B C A 6$ genes (rs10491178 $\mathrm{HR}_{\text {random }}=3.17,95 \% \mathrm{CI}$ 2.09-4.79, prandom $\left.=5.24 \times 10^{-8}\right)$. Investigating previously reported candidate genes, we found further support of a role for rs 2466571 in CD46 in FL progression. Two SNPs in $I L 8$ (rs4073 and rs2227307) previously linked with overall survival in FL, where here associated with FL progression. We also observed an association with MTHFR and FL progression but the direction was opposite of a previous report on overall survival in FL.

\section{Genome-wide association study and meta-analysis}

The increased rate of lymphoma-specific death for carriers of the A allele of rs10491178 in ABCA10 on $17 \mathrm{p} 24$ was consistent in the SCALE and UCSF cohorts, strengthening the notion of a possible causal association 
with this region. The association was independent of established prognostic risk factors (lactate dehydrogenase levels, performance status and FLIPI). ABCA10 is clustered among four other members of the ATP binding cassette $(\mathrm{ABC}) 1$ family on $17 \mathrm{q} 24$, including $A B C A 6$ harboring another five linked associated SNP markers. The $\mathrm{ABC}$ transporters - a family of proteins responsible for the movement of a wide variety of xenobiotics, lipids and metabolic products across the cell membranes - are implicated in multidrug resistance [39]. They are overexpressed in several tumor types [39], including FL [40]. The exact function of $\mathrm{ABCA} 10$ and $\mathrm{ABCA} 6$ remains to be elucidated [41], but the $\mathrm{ABCA} 3$ transporter, similar in terms of amino acid sequences and structural organization to ABCA10 and ABCA6 [41], has been shown to impede the efficacy of rituximab in aggressive B-cell lymphomas, and vincristine, anthracyclines and etopside in acute myeloid leukemia when expressed in high levels [42]. Interestingly, rs10491178 (C> T) encodes a premature stop codon (Arg (CGA) $->$ Stop (TGA)) in the $A B C A 10$ transcript (Refseq NM_080282; Ensembl ABCA10-001) [43].

We also looked up SNPs in high LD $\left(r^{2}>0.8\right)$ with rs 10491178 for potential regulatory functions in RegulomeDB, and identified another SNP rs113464685 in $A B C A 10$, in strong LD $\left(\mathrm{r}^{2}=1.00\right)$ with the top SNPs at $17 \mathrm{q} 24$, that lies in the binding site of the transcription factor paired box 5 (PAX5). PAX5 has a pivotal regulatory function in B-cell development and its aberrant expression is correlated with aggressive subsets of B-cell NHL [44]. This SNP is thus an alternative candidate to explain the observed association with the region.

The suggestive associations $\left(\mathrm{p} \leq 10^{-6}\right)$ of $\operatorname{rs} 3131729$ in the Dab, reelin signal transducer, homolog 1 (DAB1) gene on 1p32-p31, the intergenic SNP rs11932201 on chromosome 4, and rs2250066 in the kallikrein (KLK) 11 gene on 19q13, were also consistent in the SCALE and UCSF studies. The role of the DAB1 protein in the developing nervous system is well studied but for other tissues knowledge is scarce [45]. rs11932201 is flanked by two genes (long intergenic non-protein coding RNA 1085 (LINC01085) and CPEB2 antisense RNA 1 (CPEB2-AS1)) of which little is known. The $K L K$ locus on 19q13 harbors genes encoding a family of serine proteases associated with cancer risk and prognosis in several studies [46].

\section{Investigation of SNPs with reported prognostic impact in FL and established FL risk SNPs}

Upon investigating SNPs linked to FL survival in previous studies, we observed a shorter time to lymphoma progression for $\mathrm{C}$ compared to $\mathrm{A}$ allele carriers at rs2466571 in CD46 on 1q32 ( $\left.\mathrm{HR}=1.37, \mathrm{p}_{\text {trend }}=0.006\right)$, at a nominally significant level. This is consistent with a recent study of $107 \mathrm{FL}$ patients investigating event-free survival in FL $(H R=1.49$, ptrend $<0.05)[8]$. CD46 is a complement inhibitor, protecting the cell against complement-mediated lysis [47]. High expression levels of membrane-bound complement regulatory proteins such as CD46 in tumors has been shown to suppress anti-tumor T-cell responses, and may inhibit anti-tumor therapeutic activity of monoclonal antibodies, including rituximab in B-cell NHL [47].

The minor alleles of two SNPs in IL8, rs4073 and rs2227307, on 4q12-q21 were associated with longer time to progression $\left(\mathrm{rs} 4073 \mathrm{HR}_{\mathrm{A}}=0.78, \mathrm{p}=0.02 ; \mathrm{rs} 2227307\right.$ $\mathrm{HR}_{\mathrm{G}}=0.75, \mathrm{p}=0.01$ ). Two previous studies reported an association for the same two SNPs with overall survival in FL; Cerhan et al. observed a statistically significant longer overall survival with homozygosity of the same alleles ( $\mathrm{rs} 4073 \mathrm{HR}_{\mathrm{AA}}=0.47,95 \%$ CI 0.27-0.79, rs $\left.2227307 \mathrm{HR}_{\mathrm{GG}}=0.53,95 \% \mathrm{CI} 0.31-0.91\right)$ [7], whereas Aschebrook-Kilfoy et al. observed that the opposite alleles were associated with shorter overall survival $\left(\mathrm{rs} 4073 \mathrm{HR}_{\mathrm{TT}}=2.60,95 \%\right.$ CI $1.10-6.15, \quad \mathrm{rs} 2227307$ $\mathrm{HR}_{\mathrm{CC}}=2.57,95 \%$ CI 1.07-6.17) [3] (Table 1). Time to FL progression was not evaluated in these former investigations. The A allele of rs4073 has been associated with increased production of IL8 [48].

In our study, rs1801131 in MTHFR was associated with time to progression and lymphoma-specific death, and rs2069762 in the promoter of IL2 with lymphomaspecific death. However, these results are conflicting with previous reports of overall survival in FL and are therefore difficult to interpret $[7,15]$. For rs 1801274 , located in FCGR2A on 1q23, one of the two most studied variants $[5-7,10,13,14,16,17]$, we observed no associations with lymphoma progression or lymphoma-specific death. This is in line with the results of most previous studies, irrespective of how outcome was defined (progression-free $[6,10,14,16,17]$, event-free $[5,6]$ or overall survival [5,7]).

Several SNPs, including rs6457327, rs10484561 and rs2647012 in the HLA class I and II regions on 6p21, are convincingly associated with FL risk [22,24,25], suggesting an important role of this genetic region in the development of FL, and thereby possibly also in FL progression. Indeed, rs6457327 has been associated with FL transformation $[4,18]$ and overall survival $[4]$ in two previous studies (Table 1). However, we did not observe any clear associations for rs6457327, rs10484561 and rs2647012 with FL progression or lymphoma-specific death in our cohort.

\section{Strengths and weaknesses}

The strengths of the present analysis include the population-based design of the two cohorts, the large number of patients compared to previous studies, and the comprehensive evaluation of the impact of genetic 
variation in FL outcome. Population stratification was adjusted for in the GWAS and we observed little or no evidence of inflation of the statistics (lambda $\mathrm{GC}_{\mathrm{SCALE}}=$ lambda $\mathrm{GC}_{\mathrm{UCSF}}=1.00$ ) [49]. In the analysis of candidate gene variants and lymphoma progression or death, adjustment for established risk factors including age, sex, FLIPI categories and first-line rituximab resulted in marginal changes of the point estimates only.

Although candidate gene studies have obvious weaknesses compared with genome-wide studies, a strength include a higher prior probability of true findings, since they are based on a biological understanding of cancer survival pathways [50]. Still, many candidate gene findings cannot be replicated, suggesting some false-positive results in the existing literature [50]. Most of the previous studies were small $(\mathrm{N}<150)$ and the number of SNPs tested were sometimes large (up to 6679 variants), resulting in a considerable risk of chance findings. However, the present study was limited to detect moderate to strong effects $(\mathrm{HR}>1.5-2.0)$ for the investigated SNPs with acceptable probability $(\geq 0.80)$. Hence, chance findings cannot be excluded.

In the study of genetic variation and FL prognosis, different outcome measures have been used, including overall and lymphoma-specific survival and event-free survival. Progression-free survival, defined as the time from study entry to lymphoma progression or all-cause death, is generally recognized as the most valid measure in intervention studies [51]. We argue that lymphomaspecific survival/death may be superior to overall survival (but not to progression-free survival) if the study aims to test an association with lymphoma progression leading to death. In view of the high median age at diagnosis of FL and the often indolent clinical course [1], a relatively large proportion of the patients are expected to die of non-lymphoma related causes, which could dilute associations with lymphoma progression and death if overall survival is used instead. On the other hand, with the use of lymphoma-specific survival some deaths due in part to progression may have been missed potentially leading to a lower specificity. We did not observe similar results for three out of four top genetic variants and lymphoma progression, as with lymphoma-specific death, although this analysis could only be performed in a subset of the patients. Explanations for such a difference could lie in the definitions of these outcomes. While time to progression and progression-free survival primarily reflect the first part of the follow-up period, including mainly time to first-line or second-line treatment, time to lymphoma-specific death reflects a larger part of the follow-up and thus, to a higher degree, treatment response or lack of such. In the future, attempts to validate the current findings, and when comparing results between investigations, the use of similar definitions of outcome will be important for the interpretation.

\section{Conclusions}

In the present GWAS meta-analysis, there was suggestive evidence that inherited polymorphisms in the $A B C A 10$ or $A B C A 6$ genes may be associated with risk of lymphomaspecific death. In candidate-gene analysis, an association with lymphoma progression was observed for SNPs in $C D 46$ and IL8, previously linked with lymphoma progression (CD46) and overall survival (IL8). These findings need further confirmation in other independent populations or in a larger multicenter GWAS.

\section{Additional file}

\begin{abstract}
Additional file 1: Table S1. Top SNPs associated with lymphoma-specific death (prandom<5.0 x10-5) in the meta-analysis of FL patients in SCALE and UCSF ( $N=586)$. The minor allele (A1) was investigated for association. $\mathrm{A} 2=$ major allele, $\mathrm{MAF}=$ minor allele frequency, $\mathrm{NA}=$ not applicable. Table S2. Relative risk of lymphoma-specific and all-cause death in SCALE ( $=373)$, and lymphoma progression in SCALE Sweden ( $N=231)$ for the top SNPs in the pooled analysis of SCALE and UCSF. The minor allele was investigated for association. Table S3. Relative risk of lymphoma-specific death and lymphoma progression for SNPs previously associated with follicular lymphoma risk. Figure S1. Manhattan plot of the - $\log 10$ (p-values) for the association with lymphoma-specific death in SCALE (A) and UCSF (B), by chromosome and within chromosome location. The top ten SNPs in the meta-analysis are highlighted (light green). The red line marks - $\log 10(5.0 \times 10-8)$. Figure S2. QQ plot of the observed versus the expected-log(p-values) for the association with lymphoma-specific death in SCALE (A) and UCSF (B). Figure S3. Manhattan plot of the -log10(p-values) for the association with lymphoma-specific death in the meta-analysis of SCALE and UCSF. The top ten SNPs are highlighted (light green). The red line marks - $\log 10(5.0 \times 10-8)$. Figure S4. QQ plot of the observed versus the expected -log(p-values) for the association with lymphoma-specific death in the meta-analysis of SCALE and UCSF. Figure S5. Regional association plot of the top locus in the combined analysis on 17q, displaying the $p$-values of association in SCALE, extent of LD with rs10491178 as reference, relation of SNPs and genes, and estimated recombination rates at each position. Figure S6. Estimations of the power to detect associations between selected candidate SNPs and lymphoma-specific death and lymphoma progression, respectively, for different effect sizes and minor allele frequencies (MAF).
\end{abstract}

\section{Competing interests}

The authors declare that they have no competing interest.

\section{Authors' contributions}

KES was the principal investigator and takes primary responsibility for the paper; HOA, BG and MM recruited the patients; $\mathrm{HH}$ and MM provided data for the Danish cohort; JNF and JL performed and directed the laboratory work for SCALE; FB collected clinical data from medical records in Sweden; $F B, J N F, H D, R K$ and $K H$ participated in the statistical analysis; FB and KES coordinated the research; PB, LC and CS provided data for the UCSF validation cohort and performed the survival analysis in this cohort; $F B$ and KES drafted the paper; JNF, HH, HOA, BG, MM, HD, RR, KH, JL, PB, LC and CS revised the manuscript and approved of its final version before submission. All authors read and approved the final manuscript.

\section{Acknowledgements}

The study was financed by: Swedish Cancer Society 2009/1084, 2012/774 (KES); National Institutes of Health 5R01 CA69669-02 (MM, HOA) and R01CA87014, R03CA143947, R03CA150037, R01CA104682, RO1CA122663, RO1CA154643 (CFS, PMB); Lundbeck Foundation Grant R19-A2364 (HH, MM); Danish Cancer Society Grant DP 08-155 (HH, MM). FB was supported by a 
doctoral student grant and KES by the Strategic Research Program in Epidemiology at Karolinska Institutet. The financiers had no role in the study design, collection, analysis and interpretation of data, in the writing of the manuscript, or in the decision to submit the manuscript for publication.

\section{Author details \\ ${ }^{1}$ Department of Medicine Solna, Clinical Epidemiology Unit, Karolinska Institutet, Stockholm, Sweden. ${ }^{2}$ Department of Oncology, Karolinska University Hospital Solna, Stockholm, Sweden. ${ }^{3}$ Human Genetics, Genome Institute of Singapore, A*STAR, Singapore, Singapore. ${ }^{4}$ Department of Epidemiology and Biostatistics, University of California San Francisco, San Francisco, CA, USA. ${ }^{5}$ Department of Medical Epidemiology and Biostatistics, Karolinska Institutet, Stockholm, Sweden. ${ }^{6}$ Department of Epidemiology Research, Statens Serum Institut, Copenhagen, Denmark. ${ }^{7}$ Department of Immunology, Genetics and Pathology, Science for Life Laboratory, Uppsala University, Uppsala, Sweden. ${ }^{8}$ Department of Epidemiology, Harvard School of Public Health, Boston, MA, USA. ${ }^{9}$ Department of Radiology, Oncology and Radiation Science, Uppsala University, Uppsala, Sweden. ${ }^{10}$ Department of Oncology and Pathology, Karolinska Institutet, Stockholm, Sweden. " Division of National Health Surveillance and Research, Statens Serum Institut, Copenhagen, Denmark. ${ }^{12}$ Department of Epidemiology, School of Public Health, University of Alabama at Birmingham, Birmingham, AL, USA. \\ ${ }^{13}$ Department of Hematology, Karolinska University Hospital Solna, Stockholm, Sweden}

Received: 4 February 2014 Accepted: 29 September 2014 Published online: 08 October 2014

\section{References}

1. Freedman A: Follicular lymphoma: 2014 update on diagnosis and management. Am J Hematol 2014, 89(4):429-436.

2. Relander T, Johnson NA, Farinha P, Connors JM, Sehn LH, Gascoyne RD: Prognostic factors in follicular lymphoma. J Clin Oncol 2010, 28(17):2902-2913.

3. Aschebrook-Kilfoy B, Zheng T, Foss F, Ma S, Han X, Lan Q, Holford T, Chen Y, Leaderer B, Rothman N, Zhang Y: Polymorphisms in immune function genes and non-Hodgkin lymphoma survival. J Cancer Surviv 2012, 6(1):102-114.

4. Berglund M, Enblad G, Thunberg U: SNP rs6457327 is a predictor for overall survival in follicular lymphoma as well as survival after transformation. Blood 2011, 118(16):4489.

5. Carlotti E, Palumbo GA, Oldani E, Tibullo D, Salmoiraghi S, Rossi A, Golay J, Pulsoni A, Foa R, Rambaldi A: FcgammaRIIIA and FcgammaRIIA polymorphisms do not predict clinical outcome of follicular non-Hodgkin's lymphoma patients treated with sequential CHOP and rituximab. Haematologica 2007, 92(8):1127-1130.

6. Cartron G, Dacheux L, Salles G, Solal-Celigny P, Bardos P, Colombat P, Watier $\mathrm{H}$ : Therapeutic activity of humanized anti-CD20 monoclonal antibody and polymorphism in IgG Fc receptor FcgammaRIlla gene. Blood 2002, 99(3):754-758.

7. Cerhan JR, Wang S, Maurer MJ, Ansell SM, Geyer SM, Cozen W, Morton LM, Davis S, Severson RK, Rothman N, Lynch CF, Wacholder S, Chanock SJ, Habermann TM, Hartge P: Prognostic significance of host immune gene polymorphisms in follicular lymphoma survival. Blood 2007, 109(12):5439-5446

8. Charbonneau B, Maurer MJ, Fredericksen ZS, Zent CS, Link BK, Novak AJ, Ansell SM, Weiner GJ, Wang AH, Witzig TE, Dogan A, Slager SL, Habermann TM, Cerhan JR: Germline variation in complement genes and event-free survival in follicular and diffuse large B-cell lymphoma. Am J Hematol 2012, 87(9):880-885.

9. Charbonneau B, Wang AH, Maurer MJ, Asmann YW, Zent CS, Link BK, Ansell SM, Weiner GJ, Ozsan N, Feldman AL, Witzig TE, Cunningham JM, Dogan A, Habermann TM, Slager SL, Novak AJ, Cerhan JR: CXCR5 polymorphisms in non-Hodgkin lymphoma risk and prognosis. Cancer Immunol Immunother 2013, 62(9):1475-1484

10. Ghesquieres $H$, Cartron G, Seymour JF, Delfau-Larue MH, Offner F, Soubeyran P, Perrot A, Brice P, Bouabdallah R, Sonet A, Dupuis J, Casasnovas O, Catalano JV, Delmer A, Jardin F, Verney A, Dartigues P, Salles G: Clinical outcome of patients with follicular lymphoma receiving chemoimmunotherapy in the PRIMA study is not affected by FCGR3A and FCGR2A polymorphisms. Blood 2012, 120(13):2650-2657.
11. Ghielmini M, Rufibach $K$, Salles $G$, Leoncini-Franscini L, Leger-Falandry C, Cogliatti S, Fey M, Martinelli G, Stahel R, Lohri A, Ketterer N, Wernli M, Cerny T, Schmitz SF: Single agent rituximab in patients with follicular or mantle cell lymphoma: clinical and biological factors that are predictive of response and event-free survival as well as the effect of rituximab on the immune system: a study of the Swiss Group for Clinical Cancer Research (SAKK). Ann Oncol 2005, 16(10):1675-1682.

12. Gibson TM, Wang SS, Cerhan JR, Maurer MJ, Hartge P, Habermann TM, Davis S, Cozen W, Lynch CF, Severson RK, Rothman N, Chanock SJ, Morton LM: Inherited genetic variation and overall survival following follicular lymphoma. Am J Hematol 2012, 87(7):724-726.

13. Persky DO, Dornan D, Goldman BH, Braziel RM, Fisher RI, Leblanc M, Maloney DG, Press OW, Miller TP, Rimsza LM: Fc gamma receptor 3a genotype predicts overall survival in follicular lymphoma patients treated on SWOG trials with combined monoclonal antibody plus chemotherapy but not chemotherapy alone. Haematologica 2012, 97(6):937-942.

14. Racila E, Link BK, Weng WK, Witzig TE, Ansell S, Maurer MJ, Huang J, Dahle C, Halwani A, Levy R, Weiner GJ: A polymorphism in the complement component $\mathrm{C} 1 \mathrm{qA}$ correlates with prolonged response following rituximab therapy of follicular lymphoma. Clin Cancer Res 2008 14(20):6697-6703.

15. Wang SS, Maurer MJ, Morton LM, Habermann TM, Davis S, Cozen W, Lynch CF, Severson RK, Rothman N, Chanock SJ, Hartge P, Cerhan JR: Polymorphisms in DNA repair and one-carbon metabolism genes and overall survival in diffuse large B-cell lymphoma and follicular lymphoma. Leukemia 2009, 23(3):596-602.

16. Weng WK, Czerwinski D, Timmerman J, Hsu FJ, Levy R: Clinical outcome of lymphoma patients after idiotype vaccination is correlated with humoral immune response and immunoglobulin G Fc receptor genotype. J Clin Oncol 2004, 22(23):4717-4724.

17. Weng WK, Levy R: Two immunoglobulin $G$ fragment $C$ receptor polymorphisms independently predict response to rituximab in patients with follicular lymphoma. J Clin Oncol 2003, 21(21):3940-3947.

18. Wrench D, Leighton P, Skibola CF, Conde L, Cazier JB, Matthews J, labal S, Carlotti E, Bodor C, Montoto S, Calaminici M, Gribben JG, Lister TA, Fitzgibbon J: SNP rs6457327 in the HLA region on chromosome $6 p$ is predictive of the transformation of follicular lymphoma. Blood 2011, 117(11):3147-3150.

19. Gribben JG: Implications of the tumor microenvironment on survival and disease response in follicular lymphoma. Curr Opin Oncol 2010, 22(5):424-430

20. Phan VH, Tan C, Rittau A, Xu H, McLachlan AJ, Clarke SJ: An update on ethnic differences in drug metabolism and toxicity from anti-cancer drugs. Expert Opin Drug Metab Toxicol 2011, 7(11):1395-1410.

21. Bruhns P, lannascoli B, England P, Mancardi DA, Fernandez N, Jorieux S, Daeron M: Specificity and affinity of human Fcgamma receptors and their polymorphic variants for human IgG subclasses. Blood 2009, 113(16):3716-3725

22. Conde L, Halperin E, Akers NK, Brown KM, Smedby KE, Rothman N, Nieters A, Slager SL, Brooks-Wilson A, Agana L, Riby J, Liu J, Adami HO, Darabi H, Hjalgrim H, Low HQ, Humphreys K, Melbye M, Chang ET, Glimelius B, Cozen W, Davis S, Hartge P, Morton LM, Schenk M, Wang SS, Armstrong B, Kricker A, Milliken S, Purdue MP, et al: Genome-wide association study of follicular lymphoma identifies a risk locus at 6p21.32. Nat Genet 2010, 42(8):661-664.

23. Morton LM, Purdue MP, Zheng T, Wang SS, Armstrong B, Zhang Y, Menashe I, Chatterjee N, Davis S, Lan Q, Vajdic CM, Severson RK, Holford TR, Kricker A, Cerhan JR, Leaderer B, Grulich A, Yeager M, Cozen W, Hoar Zahm S, Chanock SJ, Rothman N, Hartge P: Risk of non-Hodgkin lymphoma associated with germline variation in genes that regulate the cell cycle, apoptosis, and lymphocyte development. Cancer Epidemiol Biomarkers Prev 2009, 18(4):1259-1270.

24. Skibola CF, Bracci PM, Halperin E, Conde L, Craig DW, Agana L, lyadurai K, Becker N, Brooks-Wilson A, Curry JD, Spinelli JJ, Holly EA, Riby J, Zhang L, Nieters A, Smith MT, Brown B: Genetic variants at 6p21.33 are associated with susceptibility to follicular lymphoma. Nat Genet 2009, 41(8):873-875.

25. Smedby KE, Foo JN, Skibola CF, Darabi H, Conde L, Hjalgrim H, Kumar V, Chang ET, Rothman N, Cerhan JR, Brooks-Wilson AR, Rehnberg E, Irwan ID, Ryder LP, Brown PN, Bracci PM, Agana L, Riby J, Cozen W, Davis S, Hartge P, Morton LM, Severson RK, Wang SS, Slager SL, Fredericksen ZS, Novak AJ, Kay NE, Habermann TM, Armstrong B, et al: GWAS of follicular lymphoma reveals allelic heterogeneity at $6 \mathrm{p} 21.32$ and suggests shared genetic susceptibility with diffuse large B-cell lymphoma. PLoS Genet 2011, 7(4):e1001378. 
26. Wang SS, Purdue MP, Cerhan JR, Zheng T, Menashe I, Armstrong BK, Lan Q, Hartge P, Kricker A, Zhang Y, Morton LM, Vajdic CM, Holford TR, Severson RK, Grulich A, Leaderer BP, Davis S, Cozen W, Yeager M, Chanock SJ, Chatterjee N, Rothman N: Common gene variants in the tumor necrosis factor (TNF) and TNF receptor superfamilies and NF-kB transcription factors and non-Hodgkin lymphoma risk. PLoS One 2009, 4(4):e5360.

27. Smedby KE, Hjalgrim H, Melbye M, Torrang A, Rostgaard K, Munksgaard L, Adami J, Hansen M, Porwit-MacDonald A, Jensen BA, Roos G, Pedersen BB, Sundstrom C, Glimelius B, Adami HO: Ultraviolet radiation exposure and risk of malignant lymphomas. J Natl Cancer Inst 2005, 97(3):199-209.

28. Jaffe E: Pathology and genetics of tumours of hematopoietic and lymphoid tissues. Lyon, France: IARC Press, International Agency for Research on Cancer; 2001.

29. The Swedish Cause of Death Register, The National Board of Health and Welfare, Sweden. Available at: http://www.socialstyrelsen.se/register/ dodsorsaksregistret. Accessed May 2, 2012. In.

30. D'Amore F, Christensen BE, Brincker H, Pedersen NT, Thorling K, Hastrup J, Pedersen M, Jensen MK, Johansen P, Andersen E, Bach B, Sørensen E, Danish LYFO Study Group: Clinicopathological features and prognostic factors in extranodal non-Hodgkin lymphomas. Danish LYFO Study Group. Eur J Cancer 1991, 27(10):1201-1208.

31. Purcell $S$, Neale B, Todd-Brown K, Thomas L, Ferreira MA, Bender D, Maller J, Sklar P, de Bakker PI, Daly MJ, Sham PC: PLINK: a tool set for wholegenome association and population-based linkage analyses. Am J Hum Genet 2007, 81(3):559-575.

32. Price AL, Patterson NJ, Plenge RM, Weinblatt ME, Shadick NA, Reich D: Principal components analysis corrects for stratification in genome-wide association studies. Nat Genet 2006, 38(8):904-909.

33. Skibola CF, Bracci PM, Halperin E, Nieters A, Hubbard A, Paynter RA, Skibola DR, Agana L, Becker N, Tressler P, Forrest MS, Sankararaman S, Conde L, Holly EA, Smith MT: Polymorphisms in the estrogen receptor 1 and vitamin $\mathrm{C}$ and matrix metalloproteinase gene families are associated with susceptibility to lymphoma. PLoS One 2008, 3(7):e2816.

34. DerSimonian R, Laird N: Meta-analysis in clinical trials. Control Clin Trials 1986, 7(3):177-188

35. R Development Core Team: R: A Language and Environment for Statistical Computing. Vienna, Austria: R Foundation for Statistical Computing; 2010 Retrieved from http://www.R-project.org.

36. Pruim RJ, Welch RP, Sanna S, Teslovich TM, Chines PS, Gliedt TP, Boehnke M, Abecasis GR, Willer CJ: LocusZoom: regional visualization of genome-wide association scan results. Bioinformatics 2010, 26(18):2336-2337.

37. Boyle AP, Hong EL, Hariharan M, Cheng Y, Schaub MA, Kasowski M, Karczewski KJ, Park J, Hitz BC, Weng S, Cherry JM, Snyder M: Annotation of functional variation in personal genomes using RegulomeDB. Genome Res 2012, 22(9):1790-1797.

38. Bland JM, Altman DG: Multiple significance tests: the Bonferroni method. BMJ 1995, 310(6973):170

39. Falasca M, Linton $\mathrm{KJ}$ : Investigational $A B C$ transporter inhibitors. Expert Opin Investig Drugs 2012, 21(5):657-666.

40. Yagi K, Yamamoto K, Umeda S, Abe S, Suzuki S, Onishi I, Kirimura S, Fukayama M, Arai A, Kitagawa M, Kurata M: Expression of multidrug resistance 1 gene in B-cell lymphomas: association with follicular dendritic cells. Histopathology 2013, 62(3):414-420.

41. Coleman JA, Quazi F, Molday RS: Mammalian P4-ATPases and ABC transporters and their role in phospholipid transport. Biochim Biophys Acta 2013, 1831(3):555-574.

42. Aung T, Chapuy B, Vogel D, Wenzel D, Oppermann M, Lahmann M, Weinhage T, Menck K, Hupfeld T, Koch R, Trumper L, Wulf GG: Exosomal evasion of humoral immunotherapy in aggressive B-cell lymphoma modulated by ATP-binding cassette transporter A3. Proc Natl Acad Sci U S A 2011, 108(37):15336-15341.

43. Flicek P, Amode MR, Barrell D, Beal K, Billis K, Brent S, Carvalho-Silva D, Clapham P, Coates G, Fitzgerald S, Gil L, Giron CG, Gordon L, Hourlier T, Hunt S, Johnson N, Juettemann T, Kahari AK, Keenan S, Kulesha E, Martin FJ, Maurel T, McLaren WM, Murphy DN, Nag R, Overduin B, Pignatelli M, Pritchard B, Pritchard E, Riat HS, et al: Ensembl 2014. Nucleic Acids Res 2014, 42(Database issue):D749-755

44. O'Brien P, Morin P Jr, Ouellette RJ, Robichaud GA: The Pax-5 gene: a pluripotent regulator of B-cell differentiation and cancer disease. Cancer Res 2011, 71(24):7345-7350.
45. Vazquez-Carretero MD, Garcia-Miranda P, Calonge ML, Peral MJ, Ilundain AA: Dab1 and reelin participate in a common signal pathway that controls intestinal crypt/villus unit dynamics. Biol Cell 2014, 106(3):83-96.

46. Batra J, O'Mara T, Patnala R, Lose F, Clements JA: Genetic polymorphisms in the human tissue kallikrein (KLK) locus and their implication in various malignant and non-malignant diseases. Biol Chem 2012, 393(12):1365-1390.

47. Yan J, Allendorf DJ, Li B, Yan R, Hansen R, Donev R: The role of membrane complement regulatory proteins in cancer immunotherapy. Adv Exp Med Biol 2008, 632:159-174

48. Wang N, Zhou R, Wang C, Guo X, Chen Z, Yang S, Li Y: -251 T/A polymorphism of the interleukin-8 gene and cancer risk: a HuGE review and meta-analysis based on 42 case-control studies. Mol Biol Rep 2012, 39(3):2831-2841.

49. Wang D, Sun Y, Stang P, Berlin JA, Wilcox MA, Li Q: Comparison of methods for correcting population stratification in a genome-wide association study of rheumatoid arthritis: principal-component analysis versus multidimensional scaling. BMC Proc 2009, 3(Suppl 7):S109.

50. Pasche B, Yi N: Candidate gene association studies: successes and failures. Curr Opin Genet Dev 2010, 20(3):257-261.

51. Cheson BD, Pfistner B, Juweid ME, Gascoyne RD, Specht L, Horning SJ, Coiffier B, Fisher RI, Hagenbeek A, Zucca E, Rosen ST, Stroobants S, Lister TA, Hoppe RT, Dreyling M, Tobinai K, Vose JM, Connors JM, Federico M, Diehl V: Revised response criteria for malignant lymphoma. J Clin Oncol 2007, 25(5):579-586.

\section{doi:10.1186/s12881-014-0113-6}

Cite this article as: Baecklund et al:: A comprehensive evaluation of the role of genetic variation in follicular lymphoma survival. BMC Medical Genetics 2014 15:113.

\section{Submit your next manuscript to BioMed Central and take full advantage of:}

- Convenient online submission

- Thorough peer review

- No space constraints or color figure charges

- Immediate publication on acceptance

- Inclusion in PubMed, CAS, Scopus and Google Scholar

- Research which is freely available for redistribution 